


\title{
ESPÉCIES MADEIREIRAS E ASPECTOS DA PRODUÇÃO DE PISOS EM CRUZEIRO DO SUL - ACRE, OESTE DA AMAZÔNIA
}

${ }^{1}$ Manoel Jonathan Gomes Monteiro, ${ }^{2}$ Gleisson de Oliveira Nascimento, ${ }^{3} J o s i m a r$ Batista Ferreira, ${ }^{4}$ Samara da Silva Oliveira, ${ }^{5}$ Sabrina Silva de Oliveira

1. Eng. Florestal pela Universidade Federal do Acre - UFAC.

2. Professor Mestre da Universidade Federal do Acre - UFAC - Instituto da

Biodiversidade (gleissonnascimento582@gmail.com).

3. Professor Doutor da Universidade Federal do Acre - UFAC.

4. Graduanda da Universidade Federal do Acre - UFAC.

5. Mestranda da Universidade Federal do Acre - UFAC.

Recebido em: 03/10/2016 - Aprovado em: 21/11/2016 - Publicado em: 05/12/2016 DOI: 10.18677/EnciBio_2016B_044

\begin{abstract}
RESUMO
A madeira é um material sólido que apresenta especificidades morfológicas e anatômicas que a torna um material com diversos fins de aplicações. Desta forma, seu uso abrange diversos setores. Esta pesquisa foi elaborada com objetivo de realizar um levantamento das principais espécies utilizadas para a produção de pisos no município de Cruzeiro do Sul - Acre. A cidade de Cruzeiro do Sul está localizada no oeste do estado do Acre, na região do Alto Juruá. Possui 8.779,407 $\mathrm{Km}^{2}$, com áreas de grande e baixo potencial para a atividade madeireira, sendo esta atividade composta por pequenas empresas. Neste trabalho, foram realizadas entrevistas com base em um questionário, dando ênfase nas informações gerais das empresas madeireiras e as principais espécies comercializadas. Dentre as espécies arbóreas de importância madeireira utilizadas para produção de pisos nas empresas da cidade de Cruzeiro do Sul, Acre, destacaram-se angelim, cedro, tauari, louro, milho cozido, guariúba, amargoso, jacareúba, leva tudo, miratoá, maubarana, biorana e mata-matá. Dentre estas citadas, as espécies mais procuradas são angelim, cedro e tauari como as mais utilizadas para produção de pisos na região. As empresas madeireiras são responsáveis por parte dos investimentos e geração de oportunidades no mercado de trabalho. Os produtos oriundos das mesmas e as formas de caracterizá-los são os diferenciais deste nicho de mercado, destacando-se os pisos de madeira que são produtos de consumo de grande parte da população, gerando rendas nas empresas e uma aceitação ainda considerável no mercado de Cruzeiro do Sul, Acre.
\end{abstract}

PALAVRAS-CHAVE: Espécies vegetais; Madeira; Pisos.

\section{TIMBER SPECIES AND ASPECTS OF FLOORS PRODUCTION IN CRUZEIRO DO SUL, ACRE, WESTERN AMAZON}

\footnotetext{
ABSTRACT

Wood is a solid material having specific morphological and anatomical that becomes a material with different purposes of applications. Thus, its use covers various sectors. This research was developed in order to carry out a survey of the main ENCICLOPÉDIA BIOSFERA, Centro Científico Conhecer - Goiânia, v.13 n.24; p.486 2016
} 
species used for the production of floors in the city of Cruzeiro do Sul - Acre. The city of Cruzeiro do Sul is located in the western state of Acre, in the Upper Juruá region. It has 8779.407 square kilometers, with areas of high and low potential for logging, and this activity consists of small businesses. In this work, interviews were conducted based on a questionnaire, emphasizing the general information of the logging companies and the main species marketed. Among the tree species of timber used importance for the production of floors in companies in the city of Cruzeiro do Sul, Acre, stood out angelim, cedro, tauari, louro, milho cozido, guariuba, amargoso, jacareuba, leva tudo, miratoa, maubarana, biorana and mata-mata. Among those cited, angelim stood out, cedar and tauari as the most used for the production of floors in the region. Timber companies are responsible for part of the investment and development of opportunities in the labor market. Derived products thereof and ways to characterize them are the differentials of this niche market, especially the wooden floors that are much of the population of consumer products, generating revenues in business and a still considerable acceptance in the market Cruzeiro do Sul, Acre.

KEYWORDS: Plant species; Wood; Floors.

\section{INTRODUÇÃO}

Os ecossistemas tropicais comportam grande parte da riqueza de espécies de árvores estimadas no mundo, sendo que algumas delas estão ameaçadas por uma alta taxa de desflorestamento (DUMINIL et al., 2006). Neste sentido, uma potencial ameaça para espécies de interesse comercial é a pressão exercida principalmente no que se refere à exploração de produtos florestais madeireiros e não madeireiros (RIST et al., 2012).

Nos últimos anos, observa-se o avanço das fronteiras agrícolas, juntamente com as formas de manejo dos recursos associados às mudanças climáticas indicando fortes evidências na sua contribuição para modificações na dinâmica da composição de espécies e fixação de carbono em florestas tropicais (DAVIDSON et al., 2012), sendo que um estudo recente sugere diminuição na capacidade de fixação de carbono da floresta amazônica em uma série de 10 anos de avaliação, indicando tendência decrescente na capacidade de fixação de carbono da floresta amazônica, ao passo que aumenta a biomassa morta da vegetação madura (BRIENEN et al., 2015).

Nesse sentindo, as respostas negativas da vegetação quanto ao modelo atual de exploração nos rementem à necessidade de algumas mudanças de comportamento quanto à forma atual de exploração e uso dos recursos florestais para não comprometer seu poder de resiliência. Uma forma de amenizar a utilização desordenada e a exaustão dos produtos florestais é através da gestão múltipla, que visa harmonizar as dimensões econômicas, sociais, institucionais e biofísicas relacionadas com a colheita, utilização e comercialização de uma variedade de bens e serviços oferecidos pelos produtos oriundos da floresta (HERRERO-JÁUREGUI et al., 2013).

O Brasil é um grande produtor e consumidor de produtos florestais, onde indústrias siderúrgicas, produtoras de papel, embalagens e a construção civil são altamente dependentes deste setor. Considerando que 0 país possui aproximadamente 4,8 milhões de quilômetros quadrados de floresta natural, o que representa $56 \%$ de seu território, é considerado um país com aptidão florestal (VERÍSSIMO \& PEREIRA, 2014), e este setor é considerado de grande importância para economia brasileira (SOARES et al., 2012). Assim, a agregação de valor e o 
consumo deste produto é imprescindível a elaboração de estratégias para torná-lo competitivo no mercado. A madeira possibilita a fabricação de vários produtos como, por exemplo, pisos que são utilizados para dar suporte às construções civis.

Nesta perspectiva, a demanda de piso de madeira vem crescendo a cada ano na construção civil e a matéria prima usada na confecção de outros materiais oriundos da madeira, ainda é proveniente de florestas nativas, em especial da Amazônia (SANTOS et al., 2010). Dessa forma, embora a diversidade de espécies vegetais existentes na Amazônia seja considerada abundante, a escala de produção deste produto ainda é reduzida quando comparada ao grande número de essências florestais disponíveis com potencial de uso (PADILHA et al., 2006). Assim, este trabalho foi conduzido no intuito de realizar um levantamento das principais espécies madeireiras e descrever os principais aspectos da produção de pisos no município de Cruzeiro do Sul - Acre, oeste da Amazônia visando compilar informações sobre o setor madeireiro na região.

\section{MATERIAL E MÉTODOS}

O presente estudo foi realizado no município de Cruzeiro do Sul - AC, localizado na mesorregião do Vale do Juruá. Os dados foram coletados a partir da aplicação de um questionário semi-estruturado aos proprietários das serrarias em atividade localizadas no município de Cruzeiro do Sul - Acre. A cidade de Cruzeiro do Sul está localizada no oeste do estado do Acre, Amazônia brasileira, possui $8.779,407 \mathrm{Km}^{2}$, com áreas de elevado e baixo potencial para a atividade madeireira (ZEE, 2006), sendo esta atividade representada por pequenas empresas na região.

As informações sobre os estabelecimentos entrevistados foram adquiridas com base nas sugestões de GIL (2008), a partir da aplicação de um formulário com a realização de entrevistas face a face com os proprietários das serrarias em atividade no município de Cruzeiro do Sul, Acre. O autor considera este método apropriado quando se tem a necessidade de maior aprofundamento nas respostas.

As espécies mais utilizadas pelas empresas locais para fabricação de pisos foram levantadas, sendo que a identificação foi feita de acordo com os nomes vulgares mencionados pelos entrevistados. Os nomes científicos foram identificados com base na lista de espécies florestais do Acre (ARAÚJO \& SILVA, 2000) e também através de auxílio de especialistas.

A coleta de dados foi realizada através de uma amostragem por acessibilidade por ser um método que não exige elevado nível de precisão, no qual o entrevistador seleciona os elementos da pesquisa a que têm acesso, e que melhor represente no universo pesquisado (GIL, 2008).

Dessa forma, neste trabalho, houve uma abordagem relacionada com os aspectos sociais da empresa madeireira perante o cenário do mercado local por meio da aplicação de um questionário enfatizando também as principais espécies madeireiras utilizadas para fabricação de pisos na cidade de Cruzeiro do Sul, Acre. Nesse sentido, foram entrevistadas oito empresas que atuam no setor madeireiro local, sendo então designadas como empresas "A", "B", "C", "D", "E, "F", "G" e "H".

\section{RESULTADOS E DISCUSSÕES}

De acordo com os resultados foi possível observar que a maior parte das empresas atua no setor madeireiro por um período superior a 10 anos. Identificou-se que estas empresas, geralmente estão localizadas próximas as residências dos empresários, sendo instaladas no perímetro urbano. Observou-se também que do 
total de 54 trabalhadores, ocorreu predominância de $96,49 \%$ do gênero masculino nas empresas entrevistadas (Figura 1), com jornada de trabalho de 8 horas por dia, totalizando uma jornada semanal de trabalho de 48 horas.

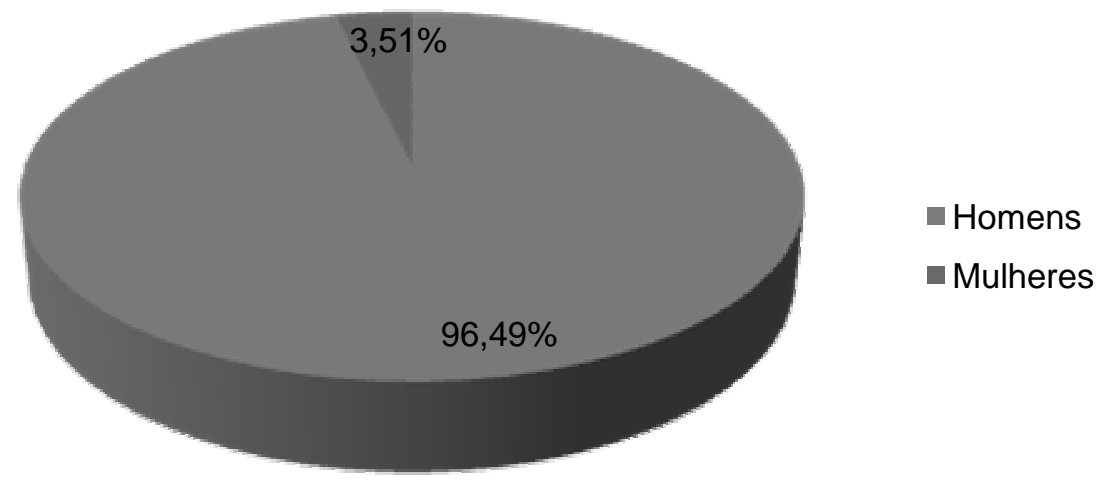

FIGURA 1 - Divisão por gênero dos funcionários das serrarias em Cruzeiro do Sul-Acre.

Apenas duas empresas das oito entrevistadas, empregavam mulheres $(3,51 \%)$ do total de 54 empregados, sendo que estas trabalhadoras atuam no setor administrativo, com a mesma carga horária que os homens. No universo amostral em que se realizou este estudo, a adoção de planos de manejo por parte dos empresários madeireiros é recente e vem sendo exigido por parte dos órgãos de fiscalização. Grande parte da matéria prima utilizada pelas empresas é proveniente de florestas naturais, embora o reconhecimento da implantação de florestas plantadas seja uma realidade.

Sabe-se que a jurisdição é clara nas exigências quanto ao manejo florestal sustentado em bases ecológicas. Na Lei no 12.651 de 25/05/2012, por exemplo, em seu artigo 33, parágrafo I, o jurista menciona que as pessoas físicas ou jurídicas que utilizam matéria prima florestal em suas atividades, devem suprir-se de recursos oriundos de florestas plantadas. Já no artigo $34^{\circ}$, a jurisprudência é contundente ao afirmar que as empresas que utilizam grande quantidade de matéria prima florestal são obrigadas a elaborar e implementar o Plano de Suprimento Sustentável - PSS, que será aprovado pelo órgão competente do SISNAMA - Sistema Nacional do Meio Ambiente (BRASIL, 2012).

Reconhecendo-se a exigência legal diante de atividades de colheita madeireira, observamos que existe certa preferência sobre determinadas espécies vegetais, o que gera oportunidades de pesquisas sobre manejo e práticas silviculturais como forma de produzir conhecimentos e técnicas que auxiliem nas melhorias deste setor. Dentre as espécies vegetais mais utilizadas para fabricação de pisos na região de estudo destacou-se o angelim (Vatairea heteroptera Ducke), seguido do cedro (Cedrela odorata L.) e tauari (Couratari macrosperma A.C.Sm.) Figura 2. 


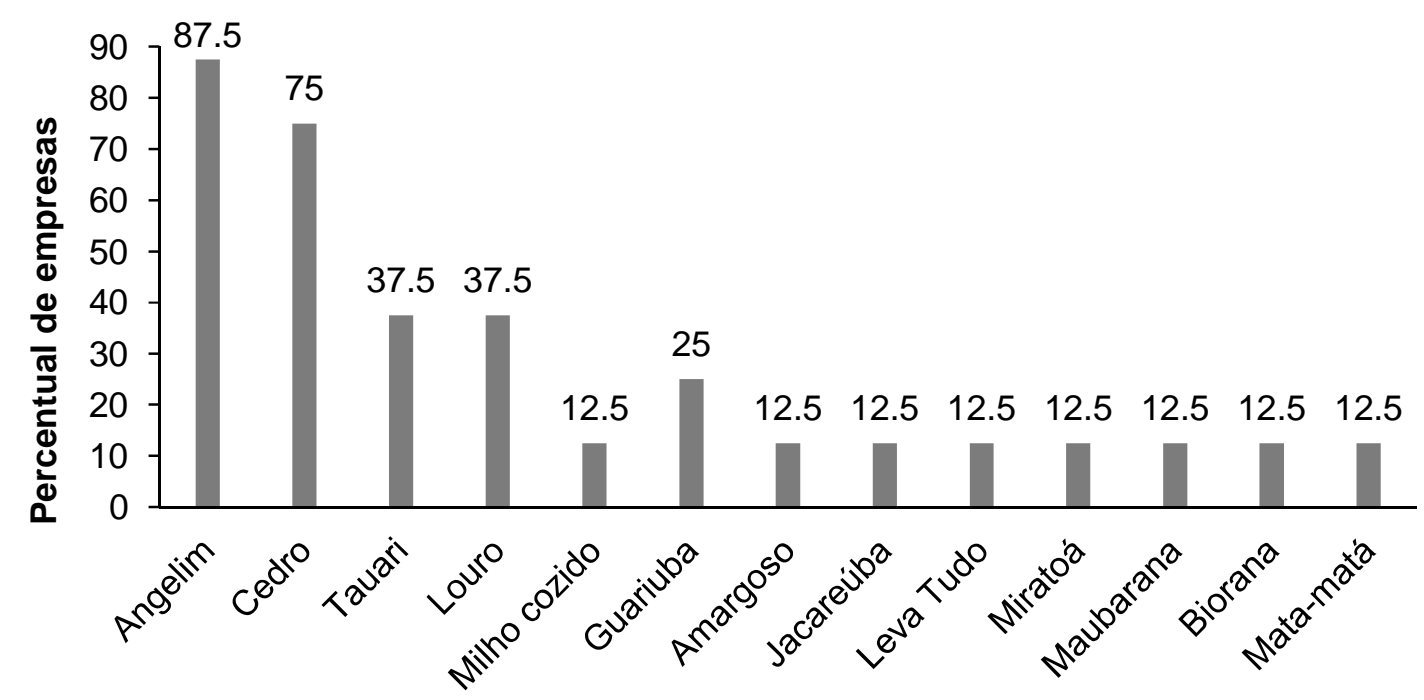

FIGURA 2 - Percentual de espécies mais utilizadas nas serrarias para construção de pisos, Cruzeiro do Sul, Acre.

A diversidade de espécies utilizadas na produção de pisos é pequena quando comparada ao grande número de essências florestais disponíveis com potencial de uso. No Estado de Mato Grosso, considerando-se período de 2004 a 2010, identificaram-se que as 10 espécies mais comercializadas em termos de volume foram Qualea sp., (cambará), Goupia glabra Aubl. (cupiúba), Erisma uncinatum Warm (cedrinho), Mezilaurus itauba (Meins.) Taub. ex Mez (itaúba), Hymenolobium sp. (angelim), Apuleia sp. (garapeira), Manilkara sp. (maçaranduba), Cordia goeldiana Huber (freijó), Dipteryx sp. (cumbarú), Trattinickia sp. (amescla), sendo que estas respondem por $88 \%$ do total de espécies madeireiras comercializadas (RIBEIRO et al., 2016).

Em estudo realizado no Estado do Acre, as principais espécies florestais manejadas legalmente entre os anos 2005 a 2012 segundo seu o vernacular e em ordem decrescente foram: cumaru-ferro (Dipteryx spp.), garapeira (Apuleia spp.), sumaúma (Ceiba spp.), açacu (Hura crepitans L.), matamatá (Eschweilera spp.), jatobá (Hymenaea spp.), tauari (Couratari spp.), manitê (Brosimun spp.), caucho (Castilla ulei Warb.), cedro (Cedrela spp.), guariúba (Clarisia racemosa Ruiz \& Pav.), faveira (Parkia spp.), copaíba (Copaifera spp.), guaribeiro (Phyllocarpus riedelii Tul.) e abiurana (Pouteria spp.) (SILVA et al., 2015).

ROBERT et al. (2012) em levantamento com o objetivo de diagnosticar a quantidade e a procedência da madeira serrada comercializada na cidade de Florianópolis, em Santa Catarina, observaram que das espécies de madeira tropical comercializadas, a espécie mais frequente encontrada nos estabelecimentos foi o angelim, seguido pelo cambará. Sendo que a madeira serrada de angelim foi encontrada em cerca de $31 \%$ dos estabelecimentos, seguida de cambará $(25 \%)$, angelim-pedra (12\%), cedrinho (10\%), garapeira (6\%), itaúba $(4,5 \%)$, jatobá $(4,5 \%)$ e outras espécies (7\%). Esses autores observaram também que $47 \%$ dos estabelecimentos avaliados usam apenas duas espécies, reforçando a falta de diversidade de espécies comercializadas na região.

Algumas espécies vegetais apresentam excelentes características tecnológicas para o aproveitamento de sua madeira, geralmente relacionadas com a 
composição química e estrutura física (GONÇALVES \& LELIS, 2012), favorecendo para sua maior procura e exploração seletiva, o que poderá ocasionar alguns efeitos negativos em suas populações, como a redução no número total de indivíduos, aumento na distância e isolamento espacial das populações remanescentes (BEM et al., 2015).

Com relação aos aspectos tecnológicos de secagem da madeira nas empresas entrevistadas, observou-se que é feita de forma natural por $87,5 \%$ das serrarias, e apenas $12,5 \%$ realizam a secagem tanto natural como artificial (em estufa) (Figura 3), o que sugere o baixo investimento em tecnologia para esta finalidade.

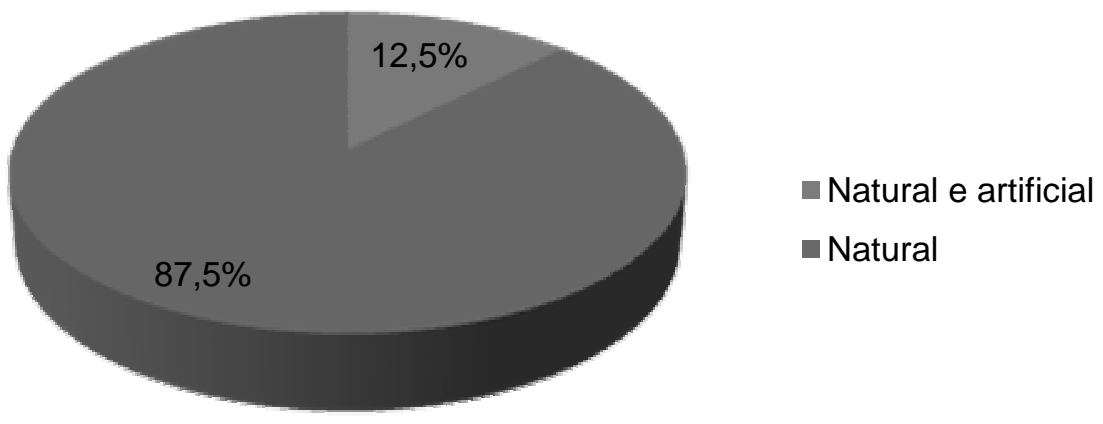

FIGURA 3 - Métodos de secagem da madeira utilizados nas serrarias de Cruzeiro do Sul, Acre.

A maneira mais prática e barata de secar madeira é àquela ao ar livre, e nesse processo de secagem, a madeira atinge a umidade de equilíbrio de forma lenta e suavemente, e o tempo de todo processo pode ser variável de acordo com as condições climáticas de cada região (BRAZ et al., 2015). Neste sentido, em um universo amostral de 12 empresas entrevistadas no município de Sinop, Mato Grosso, por meio de parâmetros qualiquantitativos, $86 \%$ realizavam a secagem ao ar livre e apenas $14 \%$ em câmaras convencionais (ANJOS et al., 2011). Ainda segundo estes autores, nas serrarias que se realiza a secagem ao ar livre, alega-se que as boas condições ambientais e a falta de preços atraentes para as madeiras secas em estufas, inviabiliza a adoção de câmaras convencionais para a secagem. Poucas empresas utilizam maquinários modernos no processo de secagem.

Com relação às condições de manuseio e de processamento de matériaprima nas indústrias madeireira de Manaus, por exemplo, de 20 movelarias pesquisadas apenas duas faziam uso de secador solar e duas das 11 serrarias utilizavam o processo de secagem em estufa (SALES-CAMPOS et al., 2000). No geral não existe seleção de espécies com características físico-químicas similares no intuito de aproximar o padrão no processo de secagem do produto.

Os pisos de madeira sob a forma de assoalho é um produto bastante comercializado na região de Cruzeiro do Sul, gerando renda para as empresas. A preferência pelo produto dá-se tanto pela situação do preço de mercado quanto pela qualidade da madeira, isso porque é alta a diversidade de espécies comercializadas 
para produção de pisos nesta região. Vale ressaltar ainda que, todas as empresas entrevistadas utilizam madeira proveniente da floresta natural, o que sugere esforços para investimento científico e tecnológico no que se refere à implantação de florestas plantadas com a finalidade de reduzir a pressão nas florestas naturais.

\section{CONCLUSÃO}

As espécies vegetais mais utilizadas para fabricação de pisos de assoalhos na região de estudo foram o cedro, angelim e tauari, sugerindo atenção especial para estas espécies vegetais de interesse econômico, uma vez que a exploração executada sem a observância de critérios técnicos, poderá ocasionar problemas futuros de oferta destes produtos na região, pois são espécies com grande aceitação no mercado por apresentarem madeira de alta durabilidade e boa trabalhabilidade.

De modo geral, as empresas madeireiras são responsáveis por parte dos investimentos e geração de oportunidades no mercado de trabalho da cidade de Cruzeiro do Sul. Os produtos oferecidos e as formas de caracterizá-los são os diferenciais deste mercado, e neste cenário destacam-se os pisos de madeira que são produtos de consumo de grande parte da população local.

\section{REFERÊNCIAS}

ANJOS, V. A.; STANGERLIN, D. M.; SANDER, A.; BOTIN, A. A.; TENUTTI, J.; SANTOS FILHO dos, N. N.; BRAGA, R. Caracterização do processo de secagem da madeira nas serrarias do município de Sinop, Mato Grosso. Ciência da Madeira, v. 02, n. 01, p. 53-63, 2011. Disponível em: <http://dx.doi.org/10.12953/21776830.v02n01a05>. Doi: 10.12953/2177-6830

ARAÚJO, H. J.; SILVA, I. G. Lista de especies florestais do Acre: ocorrencia com base em inevntarios florestais. Embrapa, 2000 (documento $n^{\circ} 48$ ), 77 p.

Disponível

em:

<https://www.agencia.cnptia.embrapa.br/Repositorio/doc48_000gebsyhpf02wx5ok0yl ax2l337wy2d.pdf>.

BEM, E. A. D.; BITTENCOURT, J. V. M.; MORAES, M. L. T. de; SEBBENN, A. M. Cenários de corte seletivo de árvores na diversidade genética e área basal de populações de Araucaria angustifolia com base em modelagem Ecogene. Scientia Forestalis, v. 43, n. 106, p. 453-466, 2015. Disponível em: $<$ http://www.ipef.br/publicacoes/scientia/leitura.asp?Article=21\&Number $=106 \& p=n>$.

BRASIL. Lei no 12.651 de 25 de Maio de 2012. Institui o novo Código Florestal Brasileiro. Brasília, $2012 . \quad$ Disponível em: <http://www.planalto.gov.br/ccivil_03/_ato2011-2014/2012/lei/l12651.htm>.

BRAZ, R. L.; DUARTE, A. P. C.; OLIVEIRA, J. T. S.; MOTTA, J. P.; ROSADO, A. M. Curva característica de secagem da madeira de Tectona grandis e Acacia mangium ao ar livre. Floresta Ambiente, v. 22, n. 1, 2015. Disponível em: <http://dx.doi.org/10.1590/2179-8087.037913>. Doi: 10.1590/2179-8087.037913

BRIENEN, R. J. W.; PHILLIPS, O. L.; FELDPAUSCH, T. R.; et al. Long-term decline of the Amazon carbon sink. Nature, v. 519, 2015. Disponível em: 
<http://www.nature.com/nature/journal/v519/n7543/full/nature14283.html>.

Doi: $10.1038 /$ nature14283

DAVIDSON, E. A.; ARAÚJO, A. C. de; ARTAXO, P.; BALCH, J. K.; BROWN, I. F.; BUSTAMANTE, M. M. C.; COE, M. T.; DEFRIES, R. S.; KELLER, M.; LONGO, M.; MUNGER, J. W.; SCHROEDER, W.; SOARES-FILHO, B. S.; SOUZA JR, C. M.; WOFSY, S. C. The Amazon basin in transition. Nature, v. 481, 2012. Disponível em: <http://www.nature.com/nature/journal/v481/n7381/full/nature10717.html>. Doi: 10.1038/nature10717

DUMINIL, J.; CARON, H.; SCOTTI, I.; CAZAL, SAINT-OMER; PETIT, R. J. Blind population genetics survey of tropical rainforest trees. Molecular Ecology, v. 15, p. 3505-3513, 2006.

Disponível

em: <https://www.ncbi.nlm.nih.gov/pubmed/17032253>. Doi: $\quad$ 10.1111/j.1365294X.2006.03040.x

GIL, A. C. Métodos e técnicas de pesquisa social. - 6. ed. - São Paulo: Atlas, 2008. $<$ http://www.uece.br/nucleodelinguasitaperi/dmdocuments/gil_metodos_de_pesquisa .pdf>.

GONÇALVES, F. G.; LELIS, R. C. C. Caracterização tecnológica da madeira de Acacia mangium Willd em plantio consorciado com Eucalipto. Floresta e Ambiente, v. 19, n. 3, p. 286-295, 2012. Disponível em: <http://dx.doi.org/10.4322/floram.2012.034>.

HERRERO-JÁUREGUI, C.; GUARIGUATA, M. R.; CÁRDENAS, D.; VILANOVA, E.; ROBLES, M.; LICONA, J. C.; NALVARTE, W. Assessing the extent of "conflict of use" in multipurpose tropical forest trees: A regional view. Journal of Environmental Management, v. 130, p. 40-47, 2013. Disponível em: < http://dx.doi.org/10.1016/j.jenvman.2013.08.044>.

PADILHA, C.; LIMA, J. T.; SILVA, J. R. M.; TRUGILHO, P. F.; ANDRADE, H. B. Avaliação da qualidade da madeira de Eucalyptus urophylla para utilização em pisos. Scientia Forestalis, Piracicaba, n. 71, p. 141-147, 2006 . < http://www.ipef.br/publicacoes/scientia/nr71/cap14.pdf>.

RIBEIRO, E. S.; SOUZA, R. A. T. M.; PAULA, M. H.; MESQUITA, R. R. S.; MOREIRA, E. L.; FAZION, H. Espécies florestais comercializadas pelo estado de Mato Grosso. Biodiversidade, v. 15, n. 2, p. 2-20, 2016. Disponível em: <http://periodicoscientificos.ufmt.br/ojs/index.php/biodiversidade/article/view/3957>.

RIST, L.; SHANLEY, P.; SUNDERLAND, T.; SHEIL, D.; NDOYE, O.; LISWANTI, N.; TIEGUHONG, J. The impacts of selective logging on non-timber forest products of livelihood importance. Forest Ecology and Management, v. 268, p. 57-69, 2012. Disponível em: <http://dx.doi.org/10.1016/j.foreco.2011.04.037>.

ROBERT, R. C. G.; SANTOS, A. S.; SANTOS, L. D.; FANTINI, A. C. Caracterização do abastecimento de madeira serrada comercializada no município de Florianópolis- 
SC. Floresta, v. 42, n. 1, p. 85-94, 2012. Disponível em: < http://dx.doi.org/10.5380/rf.v42i1.26304>.

SALES-CAMPOS, C.; ABREU, R. L. S.; VIANEZ, B. F. Indústrias madeireiras de Manaus, Amazonas, Brasil. Acta Amazônica, v. 30, n. 20, p. 319-331, 2000. Disponível em: <http://www.scielo.br/pdf/aa/v30n2/1809-4392-aa-30-2-319.pdf>.

SANTOS, I. S.; LIMA, J. T.; SILVA, J. R. M. da. Avaliação de pisos produzidos a partir de toras de 473 clones de Eucalyptus sp. com pequenos diâmetros. Cerne, v. 16, n. 4, p. 473-478, 2010. Disponível em: <http://dx.doi.org/10.1590/S010477602010000400007>. Doi: 10.1590/S0104-77602010000400007

SILVA, F. A. P. R. C.; ROBERT, R. C. G.; SANTOS, A. S.; MENDONÇA, S. D. Quantificação e avaliação das principais espécies florestais licenciadas no estado do Acre de 2005 a 2012. Floresta Ambiente, v. 22, n. 4, 2015. Disponível em: <http://dx.doi.org/10.1590/2179-8087.026212>. Doi: 10.1590/2179-8087.026212

SOARES, P. R. C.; MILAN, M.; JANKOWSKY, I. P.; MACIEL, A. J. da S.; KANIESKI, M. R. Pontos críticos do processo de produção de pisos maciços de madeira. Ciência Florestal, v. 22, n. 2, p. 353-363, 2012. Disponível em: <http://dx.doi.org/10.5902/198050985742>. Doi: 10.5902/198050985742

VERÍSSIMO, A.; PEREIRA, D. Produção na Amazonia Florestal: caracteristicas, desafios e oportudnidades. Parcerias Estratégicas, v. 19, n. 38, p. 13-44, 2014. Disponivel em:

http://seer.cgee.org.br/index.php/parcerias_estrategicas/article/viewFile/731/671>.

ZONEAMENTO ECOLÓGICO ECONÔMICO, ZEE 2006. Programa Estadual de Zoneamento Ecológico Econômico do Estado do Acre Fase II: escala 1:250.000. SEMA, Rio Branco, 356 p., 2006. Disponível em: <http://www.mma.gov.br/port/conama/processos/CD194D39/ZEE-Acrefasell_Parte1-baixareol.pdf>. 